\title{
Incomplete reossification after craniosynostosis surgery-incidence and analysis of risk factors: a clinical-radiological assessment study
}

\author{
José Luis Thenier-Villa, MD, ${ }^{1,2}$ Pablo Sanromán-Álvarez, MD, ${ }^{1,2}$ Pablo Miranda-Lloret, MD, PhD, ${ }^{1}$ \\ and María Estela Plaza Ramírez, MD, PhD'
}

1Department of Neurological Surgery, HUiP La Fe, Valencia; and ²Department of Neurosurgery, University Hospital Complex of
Vigo, Vigo, Spain

OBJECTIVE One of the principles of the surgical treatment of craniosynostosis includes the release of fused bone plates to prevent recurrence. Such bone defects require a reossification process after surgery to prevent a cosmetic problem or brain vulnerability to damage. The objective of this study is to describe and analyze the radiological and clinical evolution of bone defects after craniosynostosis.

METHODS From January 2005 to May 2016, 248 infants underwent surgical correction of craniosynostosis at HUiP La Fe Valencia; the authors analyzed data from 216 of these cases that met the inclusion criteria for this study. Various surgical techniques were used according to the age of the patient and severity of the case, including endoscopic-assisted suturectomy, open suturectomy, fronto-orbital advancement, and cranial vault remodeling. Clinical follow-up and radiological quantitative measurements in 2 periods-12-24 months and 2 years after surgery-were analyzed; 94 patients had a postoperative CT scan and were included in the radiological analysis.

RESULTS At the end of the follow-up period, 92 of 216 patients (42.59\%) showed complete closure of the bone defect, 112 patients $(51.85 \%)$ had minor bone defects, and 12 patients $(5.56 \%)$ had significant bone defects that required surgical intervention. In the multivariate analysis, age at first surgery was not significantly associated with incomplete reossification $(p=0.15)$, nor was surgical site infection $(p=0.75)$. Multivariate analysis identified area of cranial defect greater than $5 \mathrm{~cm}^{2}$ in the first CT scan as predictive of incomplete reossification $(p=0.04)$. The mean area of cranial defect in the first CT scan (12-24 months after surgery) was $3.69 \mathrm{~cm}^{2}$ in patients treated with open surgery and $7.13 \mathrm{~cm}^{2}$ in those treated with endoscopic-assisted procedures; in the multivariate analysis, type of procedure was not related to incomplete reossification $(p=0.46)$. The positive predictive value of palpation as evaluation of bone cranial defects was $50 \%$ for significant defects and $71 \%$ for minor defects.

CONCLUSIONS The incidence of cranial defects due to incomplete reossification requiring cranioplasty was $5.56 \%$ in our series. Defects greater than $5 \mathrm{~cm}^{2}$ in the first postoperative CT scan showed a positive association with incomplete reossification. Patients treated with endoscope-assisted procedures had larger defects in the initial follow-up, but the final incidence of cranial defects was not significantly different in the endoscope-assisted surgery group from that in the open surgery group.

https://thejns.org/doi/abs/10.3171/2018.2.PEDS17717

KEYWORDS craniosynostosis; incomplete reossification; craniolacunia; low-dose CT; suturectomy; craniofacial surgery

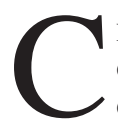

RANIOSYNOSTOSIS comprises a group of diseases characterized by the early fusion of one or more cranial sutures. Its incidence is close to 1 in 2500 individuals. ${ }^{6}$ The result of the early closure of the cranial sutures is progressive cranial deformity due to restriction and compensatory growth, with important cosmetic con- sequences. There is also a concern about the role of intracranial hypertension and cognitive impairment, as shown by the increase in studies during the last decade that refer to this aspect of the disease, ${ }^{1,3,13}$ although at present, there is no direct evidence of a causal association. ${ }^{11}$

The treatment of craniosynostosis is eminently surgi-

ABBREVIATIONS BGR = bone growth rate; MIP = maximum intensity projection; MPD = maximum perpendicular distance; ROC = receiver operating characteristic. SUBMITTED December 29, 2017. ACCEPTED February 19, 2018.

INCLUDE WHEN CITING Published online May 25, 2018; DOI: 10.3171/2018.2.PEDS17717. 
cal. Multiple surgical techniques have been used for the treatment of craniosynostosis, and each type of craniosynostosis has unique features. The spectrum of surgical techniques ranges from an open or endoscope-assisted suturectomy to cranial vault remodeling, depending on the severity of the condition and the age of the patient. ${ }^{7,15}$

One of the principles of the surgical treatment of craniosynostosis is the release of bone plates to prevent recurrent fusion. Bone defects resulting from such procedures require a reossification process after surgery to maintain the integrity of the cranial vault and avoid craniolacunia, which may represent a cosmetic problem or increase the risk of brain injury.

The incidence of cranial defects due to incomplete reossification after craniosynostosis surgery is poorly reported in the literature. A recent review reports a variable incidence of $0.5 \%$ to $18.2 \%{ }^{19}$ The aim of this study is to describe the epidemiological characteristics of incomplete reossification after craniosynostosis surgery, related factors, and reossification rates after craniosynostosis surgery.

\section{Methods \\ Study Population}

From January 2005 to May 2016, 248 patients with craniosynostosis were treated in the Department of Neurological Surgery, HUiP La Fe Valencia. We conducted a retrospective study including 216 patients.

Thirty-two patients were excluded from this study. Patients with a diagnosis of syndromic craniosynostosis and patients with coexisting central nervous system diseases, particularly hydrocephalus (including patients with a presumptive or confirmed diagnosis of hydrocephalus, ventriculomegaly, or benign enlargement of subarachnoid spaces), were excluded from the study, since they may show inherently different ossification rates. Patients treated with springs and other distractors were also excluded from our study because the surgical procedure was considered different in terms of bone removal and chance of developing secondary craniolacunia. Patients lost early in the follow-up period were also excluded.

Ninety-four patients had at least 1 low-dose 3D CT study performed postoperatively and accessible through the local picture archiving and communication system (PACS) (101 patients had a postoperative CT scan that was not recoverable in the local PACS due to storage system reorganization, and 21 patients had no postoperative CT scan).

\section{Surgical Technique}

The surgical technique for the treatment of craniosynostosis used in our center varies according to the location of the synostotic suture, the age of the patient, and the surgeon's preference. The operations performed in the cases analyzed in this study involved a variety of procedures, such as fronto-orbital advancement, cranial vault remodeling, and open and endoscope-assisted suturectomy, among others.

\section{Follow-Up Protocol}

After surgery, the patient is admitted to a pediatric in- tensive care unit for 24 hours and then discharged to the neurosurgical ward. The usual length of stay is 3 to 5 days. All patients are evaluated at 1,3, and 6 months after surgery, and then regular follow-up visits are scheduled for every 6-12 months in the outpatient office.

For the purpose of the study, follow-up was considered to have ended when a patient was discharged from the outpatient office or when a persistent cranial defect was surgically treated. The estimated time of complete bone closure was registered when such observation was specifically addressed in the clinical follow-up.

\section{Bone Defect Management}

After the 1st year of surgery, if the patient presents a bone defect smaller than $20 \mathrm{~mm}$, no intervention is recommended. If the defect is greater than $40 \mathrm{~mm}$ and persists over time, surgery is recommended. If the defect is between 20 and $40 \mathrm{~mm}$, low-dose 3D CT is performed to assess its evolution. If a defect between 20 and $40 \mathrm{~mm}$ is persistent, surgery is recommended in cases of notable cosmetic defect, critical location (i.e., over major venous sinuses), or parents' preference (Fig. 1). We usually perform the surgery when the patients are older than 4 years of age, when the chance of spontaneous reossification seems to be insignificant.

\section{Clinical and Radiological Variables of the Study}

Data pertaining to general demographic characteristics (age, sex), variables previously described as risk factors (dural tears, superficial or deep surgical site infections), and surgery-related variables (suture treated and surgical technique) were collected.

For the radiological assessment, measurements of the maximum perpendicular distance (MPD) and area of the cranial defect (A) using the maximum intensity projections (MIPs) were collected. Volume rendering was performed using VolView 3.4 (Kitware), and measurements using the software Fiji, ImageJ (see Fig. 2). The measurements were performed as follows:

- Maximum perpendicular distance (MPD) and area of cranial defect (A): MPD is defined as the maximum distance between 2 bone plates in a plane perpendicular to the suture treated. MPD1 is calculated from the first postoperative CT scan (12-24 months after surgery). MPD2 is calculated from the last postoperative CT scan (2 or more years after surgery). The area calculated on the first postoperative CT scan is designated as $\mathrm{A} 1$, and the area calculated on the last CT scan as A2.

- Bone growth rate (BGR): Bone growth rate was estimated through 2 different variables. BGRd is calculated by dividing the difference between MPD2 and MPD1 (MPD2 - MPD1) by the number of months between the 2 CT scans. BGRa is calculated by dividing the difference in area as determined on the 2 CT studies (A2 - A1) by the number of months between the studies.

\section{Statistical Analysis}

Based on clinical and radiological assessments, an analysis of risk factors for incomplete ossification was performed, using inferential statistics and survival tech- 

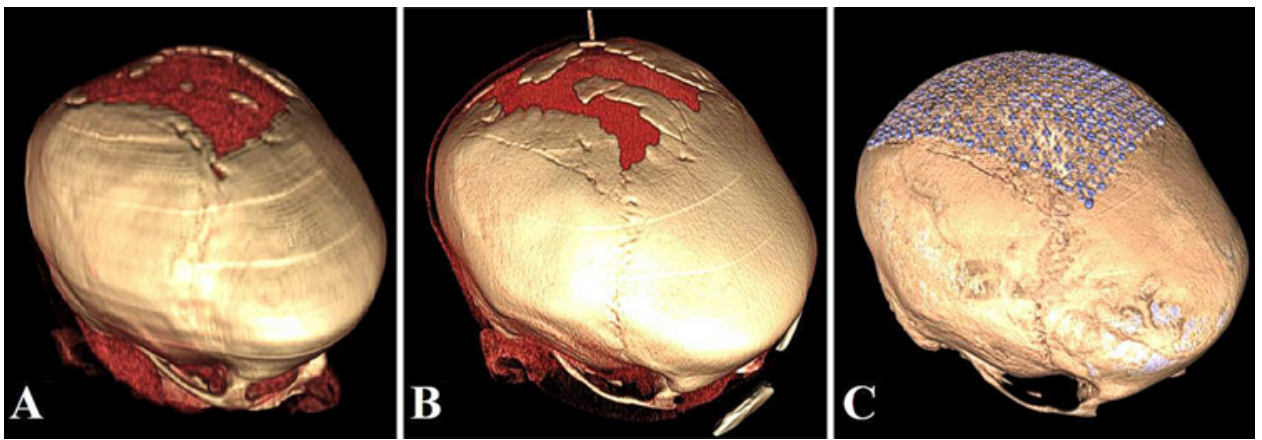

FIG. 1. Volume renderings of low-dose CT scans of a patient who underwent endoscope-assisted sagittal suturectomy at the age of 4 months. A: Study performed 5 months after surgery showing a cranial defect area of $26.4 \mathrm{~cm}^{2}$ and maximum perpendicular distance (MPD) of $48 \mathrm{~mm}$. B: Study performed 3 years later showing a persistent cranial defect $\left(\mathrm{A} 2=16.1 \mathrm{~cm}^{2}, \mathrm{MPD} 2=32\right.$ $\mathrm{mm})$. C: Postoperative CT scan obtained after treatment of the persistent defect with titanium mesh. Figure is available in color online only.

niques. The outcome variable for analyses was any incomplete reossification (including minor and major defects). Multivariate analysis was performed using the Cox proportional hazard regression model, assumptions of proportional hazards were tested using plots of log minus log (survival) functions, and no covariate violated any of the assumptions. The multivariate analysis only included patients with an accessible postoperative CT scan $(\mathrm{n}=94)$.

\section{Results}

Data from a total of 216 patients were analyzed. The patient group included 146 boys (68\%) and $70(32 \%)$ girls. Their average age at treatment was 7.91 months (range 2-96 months). The most frequent suture treated was sagittal (65.7\%), and the least frequent was lambdoid (0.9\%). Baseline characteristics of the population are summarized in Table 1.

\section{Radiological Assessment of Bone Defects}

The median MDP1 was $20 \mathrm{~mm}$ in patients who had undergone open suturectomy procedures, $18.5 \mathrm{~mm}$ in those who had undergone endoscope-assisted suturectomy procedures, $7 \mathrm{~mm}$ in those who had undergone cranial vault remodeling, and $14 \mathrm{~mm}$ in those who had undergone fronto-orbital advancement. The median MPD2 was 32 $\mathrm{mm}$ in the open suturectomy group, $12 \mathrm{~mm}$ in the endoscope-assisted suturectomy group, $9.5 \mathrm{~mm}$ in the cranial vault remodeling group, and $9 \mathrm{~mm}$ in the fronto-orbital advancement group.

The median value of the area of bone defect in the first CT scan (A1) was $2.54 \mathrm{~cm}^{2}$ in the open suturectomy group, $4.05 \mathrm{~cm}^{2}$ in the endoscope-assisted suturectomy group, $0.94 \mathrm{~cm}^{2}$ in the cranial vault remodeling group, and 1.31 $\mathrm{cm}^{2}$ in the fronto-orbital advancement group. The median value of the area of bone defect in the second $\mathrm{CT}$ scan (A2) was $10.19 \mathrm{~cm}^{2}$ in the open suturectomy group, 1.57 $\mathrm{cm}^{2}$ in the endoscope-assisted suturectomy group, 2.17 $\mathrm{cm}^{2}$ in the cranial vault remodeling group, and $0.15 \mathrm{~cm}^{2}$ in the fronto-orbital advancement group. Other measures, stratified by open versus endoscope-assisted procedure, are summarized in Table 2.
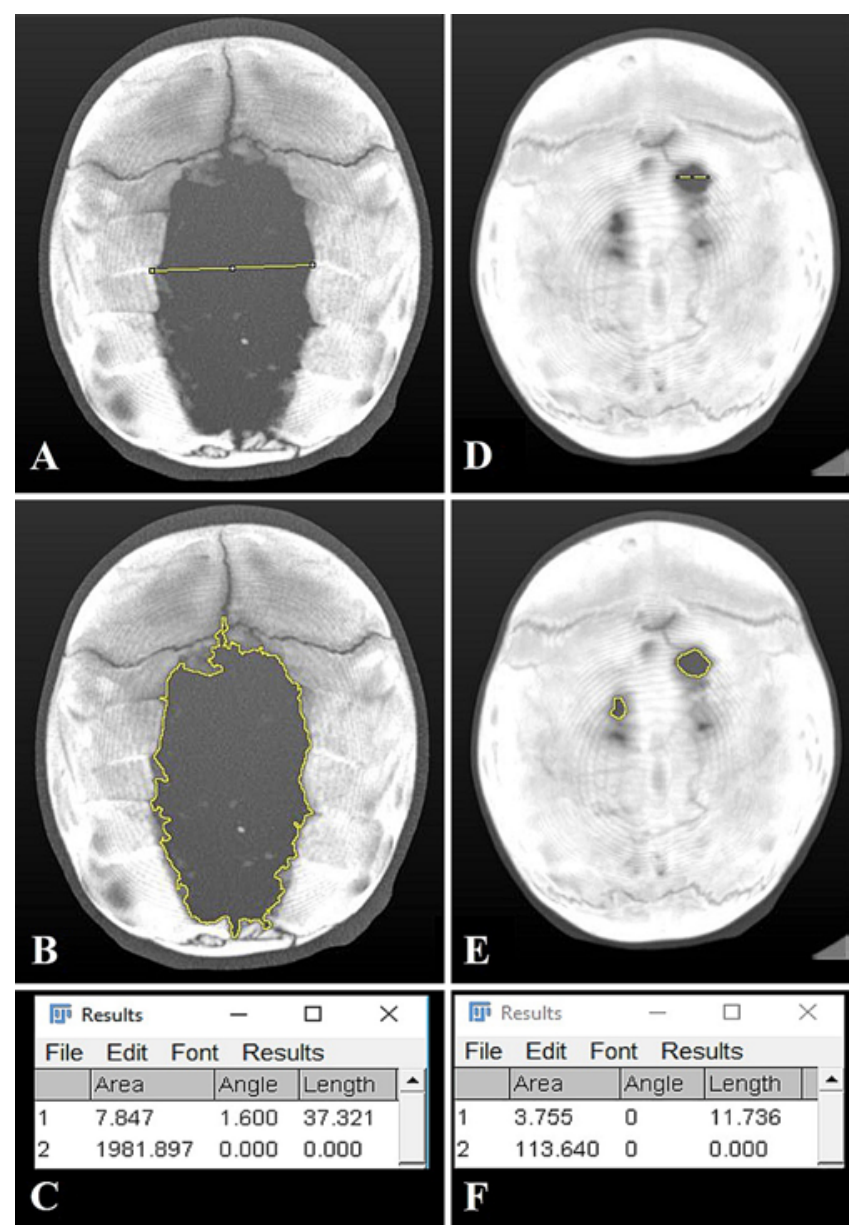

FIG. 2. Maximum intensity projections of CT scans obtained in a patient who underwent endoscope-assisted suturectomy at the age of 4 months. A: Image from postoperative CT scan showing the line of the MPD manually drawn with 2 points. B: Image from postoperative CT scan showing the semiautomatic area measurement method. C: Table of results: area $=19.81 \mathrm{~cm}^{2}$, MPD $=37 \mathrm{~mm}$. D-F: Images from CT scan performed 2 years after surgery showing MPD1 and $A 1$ measurements with the table of results $(F)$. Reossification is almost complete $(A 1=1.13$ $\mathrm{cm}^{2}$, MPD1 $=11.7 \mathrm{~mm}$ ). Figure is available in color online only. 
TABLE 1. Baseline characteristics of the patients

\begin{tabular}{|c|c|}
\hline Variable & Value \\
\hline \multicolumn{2}{|l|}{ Sex } \\
\hline Male & $146(67.6 \%)$ \\
\hline Female & $70(32.4 \%)$ \\
\hline \multicolumn{2}{|l|}{ Affected suture } \\
\hline Sagittal & $142(65.7 \%)$ \\
\hline Metopic & $32(14.8 \%)$ \\
\hline Unicoronal & $23(12.0 \%)$ \\
\hline Bicoronal & $7(3.2 \%)$ \\
\hline 2 sutures & $5(2.3 \%)$ \\
\hline More than 2 sutures & $2(0.9 \%)$ \\
\hline Lambdoid & $2(0.9 \%)$ \\
\hline \multicolumn{2}{|l|}{ Technique } \\
\hline Open procedures & $155(71.8 \%)$ \\
\hline Endoscope-assisted procedures & $61(28.2 \%)$ \\
\hline \multicolumn{2}{|l|}{ Complications } \\
\hline Surgical site infections & $15(6.9 \%)$ \\
\hline Dural tear & $8(3.7 \%)$ \\
\hline Incomplete reossification & $124(57.4 \%)$ \\
\hline Minimal bone defects & $112(51.85 \%)$ \\
\hline Significant bone defects & $12(5.56 \%)$ \\
\hline \multicolumn{2}{|l|}{ Age at surgery in mos } \\
\hline Mean & 7.9 \\
\hline Range & $2-96$ \\
\hline \multicolumn{2}{|l|}{ Follow-up time in yrs } \\
\hline Mean & 4 \\
\hline Range & $1-14$ \\
\hline
\end{tabular}

Values are numbers of cases (\%) unless otherwise indicated.

\section{Bone Defect Closure and Reossification Rate}

Regarding the clinical assessment at the end of the follow-up period, 92 patients $(42.59 \%)$ had complete closure of the bone defect, 112 patients $(51.85 \%)$ had minor bone defects, and 12 patients (5.56\%) had significant bone defects that required surgical intervention. Seventy-five percent of the patients who achieved complete ossification reached it at 36 months after surgery. Parameters of the radiological reossification rate stratified by open versus endoscope-assisted procedure are shown in Table 2.

\section{Risk Factors for Incomplete Reossification}

The survival analysis did not show significant differences in reossification rates in relation to patient $\operatorname{sex}(\mathrm{p}=$ 0.53 , log-rank test). The patients' mean age at surgery was 7.89 months in incomplete reossification cases and 8.02 months in complete reossification cases ( $p=0.52$, MannWhitney U-test). When we consider the cranioplasty as the outcome event, age at surgery showed no statistically significant difference ( $p=0.92$, Mann-Whitney U-test).

Surgical site infection, with an incidence of $6.9 \%$, similar to previous studies, ${ }^{4,21,25,26}$ was not associated with incomplete ossification in survival analysis $(p=0.33$, log-rank test) or univariate analysis $(\mathrm{p}=0.45$, chi-square test). Although patients suffering an infection showed an initial delay in the process of ossification (the first case of complete reossification was noticed at 45 months), the final incidence of bone closure was similar in cases with and without infection (57\% vs 53\%). There was also no significant difference in the frequency of incomplete reossification between patients with and without intraoperative dural tears ( $\mathrm{p}=0.77$, chi-square test). Regarding the treated suture, we observed a higher rate of complete reossification (0.9) in the metopic suture $(\mathrm{p}=0.008, \log$-rank test) in the survival analysis.

Patient age at surgery was significantly different between patients operated on with endoscope-assisted and open procedures (mean 3.44 vs 9.76 months, respectively, $\mathrm{p}=0.001$, Mann-Whitney U-test). Rates of incomplete reossification were higher in patients undergoing open procedures than in those treated by endoscope-assisted surgery in the univariate analysis ( $74 \%$ vs $49 \%$, respectively, $\mathrm{p}=0.01$, logistic regression).

The mean area of cranial defect in the first postoperative CT scan (12-24 months) was $3.69 \mathrm{~cm}^{2}$ in patients who had undergone open procedures and $7.13 \mathrm{~cm}^{2}$ in those who had undergone endoscope-assisted procedures $(\mathrm{p}=$ 0.646, Mann-Whitney U-test). In the last CT scan (after 2 years), it was 2.89 and $10.73 \mathrm{~cm}^{2}$ in the endoscope-as-

TABLE 2. Radiological measurements after craniosynostosis surgery

\begin{tabular}{|c|c|c|c|}
\hline Parameter & Open Procedures & Endoscope-Assisted Procedures & Overall \\
\hline MPD1 in mm & $20.6(10.1)$ & $18.9(12.8)$ & $14(11.14)$ \\
\hline MPD2 in mm & $31.8(22)$ & $15.2(12.4)$ & $17.8(16.9)$ \\
\hline $\mathrm{A} 1$ in $\mathrm{cm}^{2}$ & $3.69(3.11)$ & $7.13(8.8)$ & $3.8(6)$ \\
\hline $\mathrm{A} 2$ in $\mathrm{cm}^{2}$ & $10.73(9.4)$ & $2.89(4.4)$ & $5.4(8.1)$ \\
\hline BGRd in $\mathrm{mm} / \mathrm{mo}$ & $0.37(0.38)$ & $0.84(0.77)$ & $0.29(0.32)$ \\
\hline BGRa in $\mathrm{cm}^{2} / \mathrm{mo}$ & $0.10(0.05)$ & $0.40(0.36)$ & $0.67(0.67)$ \\
\hline
\end{tabular}

$\mathrm{A} 1=$ area of cranial defect calculated on the first postoperative $\mathrm{CT}$ scan; $\mathrm{A} 2=$ area of cranial defect calculated on the last $\mathrm{CT}$ scan; $\mathrm{BGR}=$ bone growth rate; $\mathrm{BGRa}=$ difference in area between the 2 CT scans (A2 - A1) divided by the number of months; BGRd = difference between MPD2 and MPD1 (MPD2 - MPD1) divided by the number of months between the $2 \mathrm{CT}$ scans; $\mathrm{MPD}=$ maximum perpendicular distance; $\mathrm{MPD} 1=\mathrm{MPD}$ calculated from the first postoperative $\mathrm{CT}$ scan; MPD2 = MPD calculated from the last postoperative CT scan.

All values are mean (SD). 


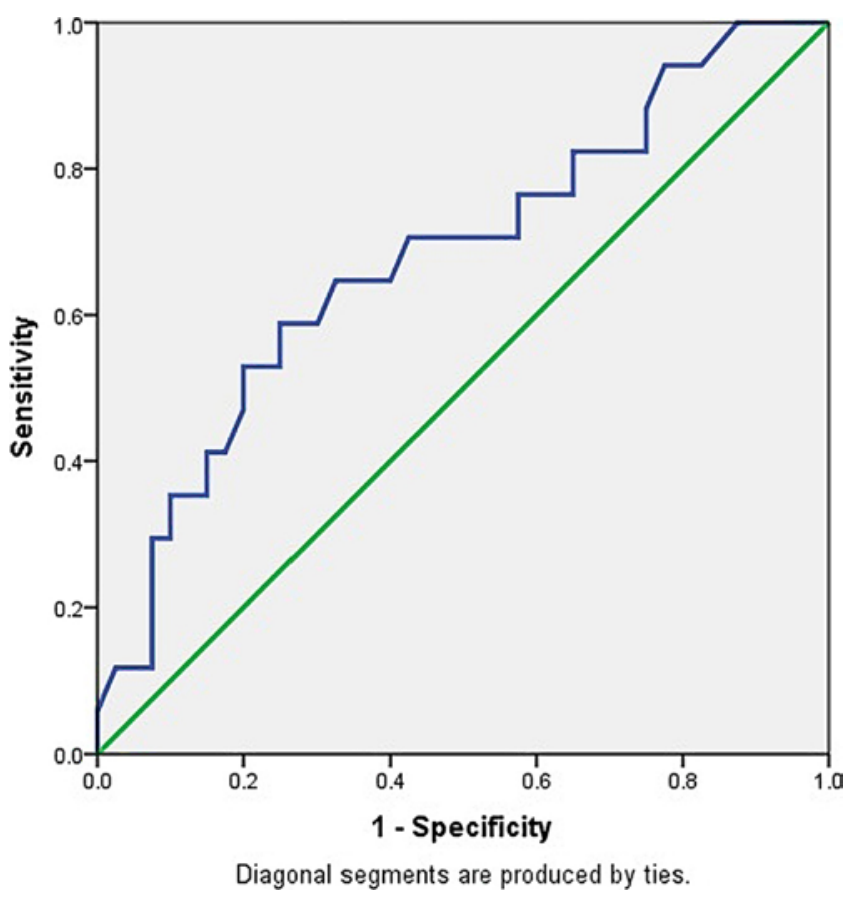

FIG. 3. ROC curve: bone defect area on first CT scan and incomplete reossification; area under the curve $=0.68$. Figure is available in color online only.

sisted and open procedure groups, respectively $(\mathrm{p}=0.419$, Mann-Whitney U-test). The mean bone growth rate in maximum perpendicular distance (BGRd) was $0.37 \mathrm{~mm} /$ month in the open procedure group and $0.84 \mathrm{~mm} / \mathrm{month}$ in the endoscope-assisted procedure group $(\mathrm{p}=0.52$, Mann-Whitney U-test). The mean BGRa was 0.10 and $0.40 \mathrm{~cm}^{2} /$ month, respectively, in the 2 groups $(\mathrm{p}=0.10$, Mann-Whitney U-test).

Regarding the radiological variables studied, MPD1 was not significantly associated with complete reossification ( $p=0.4$, Mann-Whitney U-test), whereas A1 was significantly higher in the patients with incomplete reossification $\left(6.22 \mathrm{~cm}^{2}\right.$ and $2.73 \mathrm{~cm}^{2}$ in the incomplete and complete reossification groups, respectively, $\mathrm{p}=0.04$, t-test).

When this association was evaluated with receiver operating characteristic (ROC) curves to determine the most appropriate cutoff value (Fig. 3), we established a cut-off point of $5 \mathrm{~cm}^{2}$ (sensitivity $47 \%$, specificity $80 \%$ ); for sub- sequent analysis, this cutoff was selected because in our clinical scenario the lowest false-positive rate seemed most important to avoid unnecessary surgery.

Both MPD2 (mean $26.8 \mathrm{~mm}$ vs $7.2 \mathrm{~mm}, \mathrm{p}=0.01$, Mann-Whitney U-test) and A2 area $\left(9 \mathrm{~cm}^{2}\right.$ vs $1 \mathrm{~cm}^{2}, \mathrm{p}=$ 0.001, Mann-Whitney U-test) were significantly higher in the incomplete reossification group than in the complete reossification group.

The bone growth rate in the maximum perpendicular distance was higher in patients with complete reossification $(0.88 \mathrm{~mm} /$ month vs $0.11 \mathrm{~mm} / \mathrm{month}$ in those with incomplete reossification, $\mathrm{p}=0.48$, Mann-Whitney U-test). On the other hand, the reossification rate per area (BGRa) was not significantly different in the 2 groups $\left(0.34 \mathrm{~cm}^{2} /\right.$ month vs $0.15 \mathrm{~cm}^{2} / \mathrm{month}$ in the complete and incomplete reossification groups, respectively, $\mathrm{p}=0.049$, Mann-Whitney U-test).

Multivariate analysis identified only the area of cranial defect in the first CT scan (12-24 months) as predictive of incomplete reossification at the end of the follow-up period (see Table 3 and Fig. 4).

\section{Clinical-Radiological Correlation in the Assessment of Cranial Defects}

To check the accuracy of physical examination in the assessment of postoperative bone defects, we compared the presence of bone defects in the medical records with the findings on analysis of CT scans. In the 1-year postoperative follow-up visit, among patients clinically evaluated as having no cranial defects, $\mathbf{7 7 . 2 7 \%}$ had radiological defects of less than $10 \mathrm{~mm}$, and $13 \%$ showed defects between 10 and $20 \mathrm{~mm}$. Among patients who were found to have small and insignificant defects based on clinical evaluation, $62 \%$ had defects of less than $20 \mathrm{~mm}$ in maximum perpendicular distance. Regarding patients evaluated clinically as showing intermediate defects, $71.43 \%$ had radiological defects of 20-40 $\mathrm{mm}$ in maximum perpendicular distance. Finally, among patients with defects described as significant, $50 \%$ had radiological defects with maximum distances greater than $40 \mathrm{~mm}$.

\section{Discussion}

\section{Radiological Assessment of Bone Defects}

The present study presents quantitative radiological measurements based on CT scans. Recently some studies have used quantitative methods to assess bone defects in craniosynostosis. ${ }^{2,24}$ Given that it is an easy technique

TABLE 3. Multivariate analysis for incomplete reossification

\begin{tabular}{|c|c|c|c|c|c|c|}
\hline \multirow[b]{2}{*}{ Factor } & \multicolumn{3}{|c|}{ Univariate Analysis } & \multicolumn{3}{|c|}{ Multivariate Analysis } \\
\hline & HR & $95 \% \mathrm{Cl}$ & p Value & $\mathrm{HR}$ & $95 \% \mathrm{Cl}$ & $p$ Value \\
\hline Infection & 1.52 & $0.50-4.62$ & 0.45 & 1.57 & $0.89-28.01$ & 0.75 \\
\hline Lower age at surgery & 1.01 & $0.97-1.03$ & 0.91 & 5.34 & $0.45-52.46$ & 0.15 \\
\hline Area $>5 \mathrm{~cm}^{2}$ & 3.55 & $1.04-12.13$ & 0.04 & 4.57 & $1.02-20.44$ & 0.04 \\
\hline Open procedure & 2.82 & $1.3-6.13$ & 0.01 & 1.73 & $0.39-7.56$ & 0.46 \\
\hline Dural tear & 1.23 & $0.28-5.29$ & 0.77 & 1.74 & $0.12-24.16$ & 0.12 \\
\hline
\end{tabular}



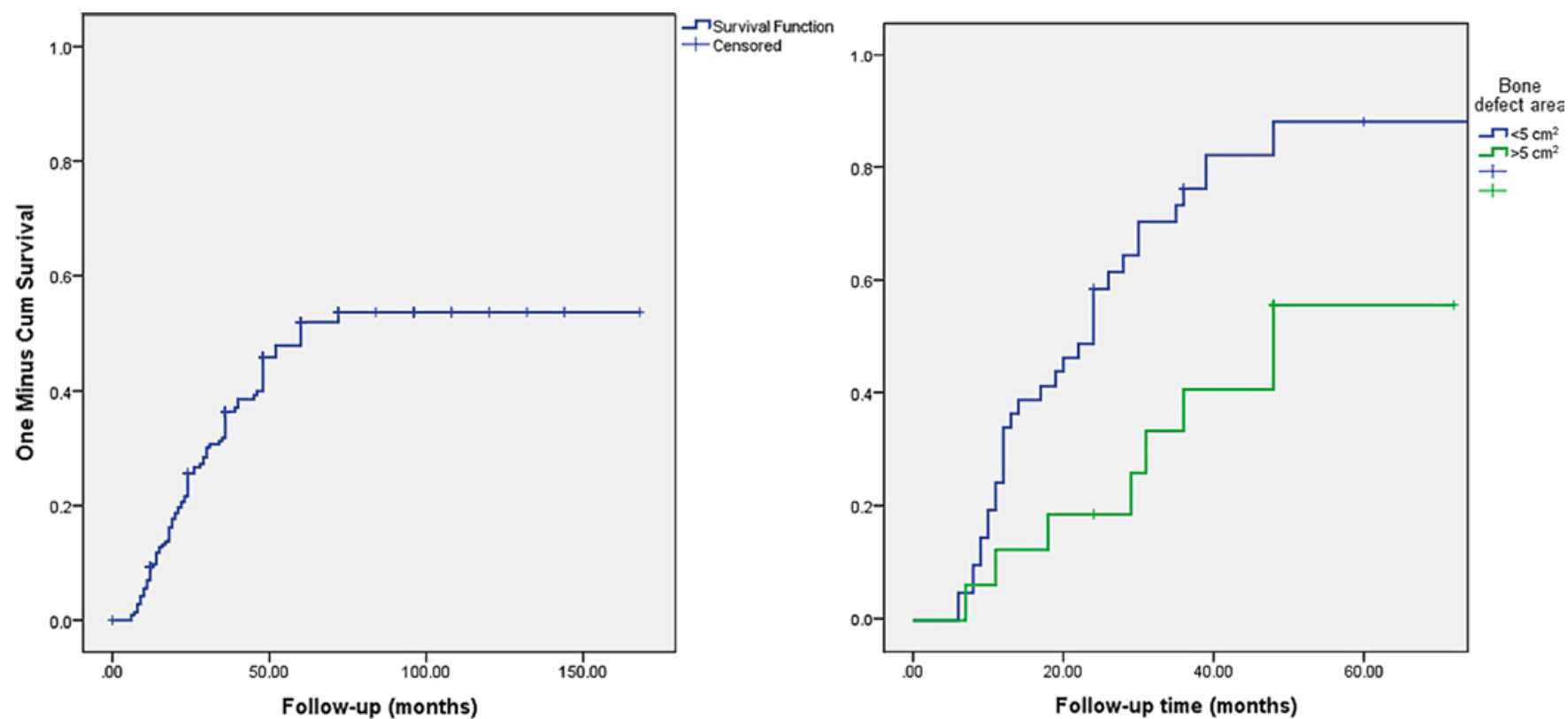

FIG. 4. Kaplan-Meier plots of bone defect closure after craniosynostosis surgery. Left: Overall survival plot. Right: Survival stratified by area of bone defect on first CT. Cum = cumulative. Figure is available in color online only.

to develop with current image visualization software, we think that all studies of craniosynostosis surgery outcomes may benefit from including this type of assessment as part of the analysis of the results.

\section{Bone Defect Closure and Reossification Rate}

Paige et al. suggested that no defect present after 1 year will completely close. ${ }^{20}$ The authors analyzed in their series only fronto-orbital advancement procedures, which in our study were associated with a lower rate of reossification; the surgical age of the patients was significantly higher in their study than in ours (median 12 vs 5 months), and their evaluation method was palpation only. Hassler and Zentner, ${ }^{10}$ reported their experience in 60 patients with sagittal synostosis treated with radical craniectomy. While children under 6 months of age at surgery showed complete ossification at the age of 1 year, those operated on between 7 and 12 months of age showed longer ossification times and reached complete closure of the defects at a maximum of 18 months, although quantitative measurements were not described in the cited study.

In contrast to the previous studies, a recent quantitative radiological study showed that the fractional healing rate ranges from $58 \%$ to $71 \% 2$ years after surgery. ${ }^{2}$ In our study, $75 \%$ of the patients who achieved complete ossification reached it at 36 months after surgery. This finding is consistent with the previous study, and given these data, we suggest that a defect should not be considered to be persistent and require surgical treatment before 3 years of postoperative follow-up.

Several methods of cranial reconstruction were used throughout our series. The most commonly used techniques included bioabsorbable plates (Lactosorb, Walter Lorenz Surgical) embedded in silicate-substituted calcium phosphate (Actifuse, ApaTech) and hydroxyapatite cement
(Mimix, Walter Lorenz Surgical, Inc.) or standard titanium mesh fixed with screws. Although autologous bone is the ideal material for reconstructing cranial defects, it frequently requires performing additional osteotomies. Split calvarial bone graft has traditionally been limited to patients older than 6 years, ${ }^{14}$ although some recent studies suggest this technique could be employed in children as young as 2 months; ${ }^{27}$ this technique is now our preferred option when feasible, but it was not a common tool in our surgical armamentarium when most of these patients were treated. On the other hand, alloplastic cranioplasty is an easy and short procedure, is relatively inexpensive, and provides good cosmetic results.

\section{Risk Factors for Incomplete Reossification}

Previous studies have identified a number of risk factors for incomplete reossification, including age, dural tears, infections, and the extent of bone defect after surgery. ${ }^{19}$ In our study, only the area in the first CT scan (12-24 months) was predictive of incomplete ossification; particularly defects greater than $5 \mathrm{~cm}^{2}$ showed a significant association with persistent bone defects. Infection cases showed a delay in the onset of the survival curve for complete reossification, but the final rate was not different in this group of patients.

Animal studies have suggested that the process of reossification relies on the dura mater ${ }^{12,18,23}$ and that younger animals have a higher rate of complete reossification. It seems that reossification is dependent on a critical period of development and there is no linear relationship between both variables after this period. ${ }^{9}$ These findings have not been replicated systematically in humans. Other studies have established different age cutoff points, which range between 9 and 24 months,,, 22 as a risk factor for incomplete reossification after craniosynostosis surgery. In our 
study, age at surgery was not identified as a predictive variable in multivariate analysis.

Because some patients had 2 or more postoperative CT scans, we were able to evaluate the bone growth rate of the cranial defects. BGR in maximum perpendicular distance but not BGR in area was related to persistent cranial defects in our study. From these data, we hypothesize that the maximum distance between 2 bone plates perpendicular to the major axis of the defect best represents the chance of reossification of the bone defect. On the other hand, the defect area in the first radiological study represents the radicality of bone resection, and this variable could be related to persistent cranial defects as previously suggested. ${ }^{17}$

In endoscope-assisted procedures the area of bone resection was slightly large compared to open procedures, but the rate of incomplete reossification was similar. While the univariate analysis showed a lower rate of incomplete reossification in endoscope-assisted procedures, multivariate analysis including age and cranial defect area after surgery showed that endoscope-assisted procedures were not related to incomplete reossification. In our series, patients treated with endoscope-assisted procedures were operated on at a significantly younger age than those treated with open procedures. This fact may explain why endoscopically treated patients did not develop higher rates of defects even though MPD1 and A1 were higher than in patients treated with standard open procedures. The bone growth rate may also have a role in this compensation since it was slightly higher in patients treated with endoscope-assisted procedures in our series.

\section{Clinical-Radiological Correlation in the Assessment of Cranial Defects}

In previous studies, the method of assessment of cranial defects varied between different centers. CT scan measurement is an objective method for evaluating bone defects. It has the disadvantage of requiring ionizing radiation, and sedation is also required in some patients. With the advent of low-radiation dose techniques, good-quality images can be obtained with a low risk of radiation injury, making CT scan useful for the diagnosis and follow-up of these patients. ${ }^{16}$

On the other hand, clinical assessment by palpation is practical and fast and can be easily performed in outpatient visits; however, it is a subjective test, so bone tissue can also be mistaken by the texture of other tissues, including the skin, the surgical scar, and even the dura mater. Some authors argue that defects not assessed by palpation are considered irrelevant. ${ }^{8,20}$ In our study, the positive predictive value of palpation ranged from $50 \%$ to $77 \%$, demonstrating its imperfection compared to objective methods; this difference becomes especially relevant in the group of patients with defects between 20 and $40 \mathrm{~mm}$, in whom an objective radiological assessment of the defect would entail a change in the conservative perspective of management.

\section{Limitations}

This study has limitations inherent to its retrospective nature. First, nearly half of the CT scans (those performed before 2009) were not stored in the local PACS and we were not able to include them in the radiological analysis. However, since the reason was a storage system reorganization, and this loss of information was unrelated to clinical events, we do not consider this a source of bias in our results. Second, the first postoperative scan was usually performed between the 1st and 2nd year after surgery and may contain some growth component, making it difficult to assess whether the natural bone growth rate during the 1 st year has a major role in the complete reossification process. Nonetheless, the calculated BGR with the available studies was similar in patients with defects larger than 5 $\mathrm{cm}^{2}$, suggesting that this component is insignificant. Most of these limitations can be solved with a prospective study.

\section{Conclusions}

The incidence of cranial defects due to incomplete reossification requiring cranioplasty in our series was $5.56 \%$. The only factor related to incomplete reossification was the area of the defect $\left(>5 \mathrm{~cm}^{2}\right)$ in the first postoperative radiological study (12-24 months). The positive predictive value of palpation as evaluation of bone cranial defects was $50 \%$ and $71 \%$ for significant and minor defects, respectively.

Endoscope-assisted procedures were performed in younger patients and patients in the endoscope-assisted surgery group had larger defects in the initial follow-up, but the final incidence of cranial defects in patients treated with endoscope-assisted procedures was not significantly different from that in patients treated at older ages with open surgical procedures.

\section{Acknowledgments}

We thank our colleagues, medical residents, surgeons, intensivists, nurses, and all the personnel of Hospital Universitari I Politecnic $\mathrm{La} \mathrm{Fe}$, for their unwavering commitment to the care of our most vulnerable patients. Corresponding authorities in both hospitals, Hospital Universitari I Politecnic La Fe and Complexo Hospitalario Universitario de Vigo, allowed us to share our clinical experience as an example of friendly interinstitutional cooperation.

The first author of this study, J. L. Thenier-Villa, gratefully acknowledges Dra. Maria Regueira Portas and Dr. Antonio Viladrich Carreira, pediatric neurosurgeons of the Complexo Hospitalario Universitario de Vigo, for their valuable guidance and support. The surgical training under their supervision was key in developing this article.

\section{References}

1. Beez T, O'Kane R, Piper I, Koppel D, Sangra M: Telemetric intracranial pressure monitoring in syndromic craniosynostosis. J Craniofac Surg 27:1032-1034, 2016

2. Chia-Chi T, Shapiro LG, Hopper RA, Halen JV: Pediatric cranial defect surface analysis for craniosynostosis postoperation CT images, in 2008 5th IEEE International Symposium on Biomedical Imaging: From Nano to Macro. Piscataway, NJ: IEEE, 2008, pp 620-623

3. Eide PK, Helseth E, Due-Tønnessen B, Lundar T: Assessment of continuous intracranial pressure recordings in childhood craniosynostosis. Pediatr Neurosurg 37:310-320, 2002

4. Esparza J, Hinojosa J, García-Recuero I, Romance A, Pascual B, Martínez de Aragón A: Surgical treatment of isolated and syndromic craniosynostosis. Results and complications in 283 consecutive cases. Neurocirugia (Astur) 19:509-529, 2008 
5. Gao LL, Rogers GF, Clune JE, Proctor MR, Meara JG, Mulliken JB, et al: Autologous cranial particulate bone grafting reduces the frequency of osseous defects after cranial expansion. J Craniofac Surg 21:318-322, 2010

6. Garza RM, Khosla RK: Nonsyndromic craniosynostosis. Semin Plast Surg 26:53-63, 2012

7. Gerety PA, Basta MN, Fischer JP, Taylor JA: Operative management of nonsyndromic sagittal synostosis: a head-to-head meta-analysis of outcomes comparing 3 techniques. J Craniofac Surg 26:1251-1257, 2015

8. Greene AK, Mulliken JB, Proctor MR, Rogers GF: Primary grafting with autologous cranial particulate bone prevents osseous defects following fronto-orbital advancement. Plast Reconstr Surg 120:1603-1611, 2007

9. Hassanein AH, Couto RA, Nedder A, Zielins ER, Greene AK: Critical-size defect ossification: effect of leporid age in a cranioplasty model. J Craniofac Surg 22:2341-2343, 2011

10. Hassler W, Zentner J: Radical osteoclastic craniectomy in sagittal synostosis. Neurosurgery 27:539-543, 1990

11. Hayward R, Britto J, Dunaway D, Jeelani O: Connecting raised intracranial pressure and cognitive delay in craniosynostosis: many assumptions, little evidence. J Neurosurg Pediatr 18:242-250, 2016

12. Hobar PC, Schreiber JS, McCarthy JG, Thomas PA: The role of the dura in cranial bone regeneration in the immature animal. Plast Reconstr Surg 92:405-410, 1993

13. Inagaki T, Kyutoku S, Seno T, Kawaguchi T, Yamahara T, Oshige H, et al: The intracranial pressure of the patients with mild form of craniosynostosis. Childs Nerv Syst 23:14551459,2007

14. Inoue A, Satoh S, Sekiguchi K, Ibuchi Y, Katoh S, Ota K, et al: Cranioplasty with split-thickness calvarial bone. Neurol Med Chir (Tokyo) 35:804-807, 1995

15. Jimenez DF, Barone CM: Endoscopic techniques for craniosynostosis. Atlas Oral Maxillofac Surg Clin North Am 18:93-107, 2010

16. Montoya JC, Eckel LJ, DeLone DR, Kotsenas AL, Diehn FE, Yu L, et al: Low-dose CT for craniosynostosis: preserving diagnostic benefit with substantial radiation dose reduction. AJNR Am J Neuroradiol 38:672-677, 2017

17. Moss SD, Joganic E, Manwaring KH, Beals SP: Transplanted demineralized bone graft in cranial reconstructive surgery. Pediatr Neurosurg 23:199-205, 1995

18. Mossaz CF, Kokich VG: Redevelopment of the calvaria after partial craniectomy in growing rabbits: the effect of altering dural continuity. Acta Anat (Basel) 109:321-331, 1981

19. Noordzij N, Brouwer R, van der Horst C: Incomplete reossification after craniosynostosis surgery. J Craniofac Surg 27:e105-e108, 2016

20. Paige KT, Vega SJ, Kelly CP, Bartlett SP, Zakai E, Jawad AF, et al: Age-dependent closure of bony defects after frontal orbital advancement. Plast Reconstr Surg 118:977-984, 2006
21. Parikh RP, Farber SJ, Nguyen D, Skolnick GB, Patel K, Woo AS: Risk factors for postoperative complications after surgical correction of craniosynostosis: a nationwide analysis of 1357 intracranial procedures. Plast Reconstr Surg 136 (4 Suppl):40, 2015

22. Pattisapu JV, Gegg CA, Olavarria G, Johnson KK, Ruiz RL, Costello BJ: Craniosynostosis: diagnosis and surgical management. Atlas Oral Maxillofac Surg Clin North Am 18:77-91, 2010

23. Reid CA, McCarthy JG, Kolber AB: A study of regeneration in parietal bone defects in rabbits. Plast Reconstr Surg 67:591-596, 1981

24. Savolainen M, Ritvanen A, Hukki J, Vuola P, Telkkä J, Leikola J: Promoting ossification of calvarial defects in craniosynostosis surgery by demineralized bone plate and bone dust in different age groups. J Plast Reconstr Aesthet Surg 70:110-119, 2017

25. Shastin D, Peacock S, Guruswamy V, Kapetanstrataki M, Bonthron DT, Bellew M, et al: A proposal for a new classification of complications in craniosynostosis surgery. J Neurosurg Pediatr 19:675-683, 2017

26. Tahiri Y, Paliga JT, Wes AM, Whitaker LA, Bartlett SP, Taylor JA: Perioperative complications associated with intracranial procedures in patients with nonsyndromic single-suture craniosynostosis. J Craniofac Surg 26:118-123, 2015

27. Vercler CJ, Sugg KB, Buchman SR: Split cranial bone grafting in children younger than 3 years old: debunking a surgical myth. Plast Reconstr Surg 133:822e-827e, 2014

\section{Disclosures}

The authors report no conflict of interest concerning the materials or methods used in this study or the findings specified in this paper.

\section{Author Contributions}

Conception and design: all authors. Acquisition of data: ThenierVilla, Sanromán-Álvarez. Analysis and interpretation of data: all authors. Drafting the article: all authors. Critically revising the article: all authors. Reviewed submitted version of manuscript: all authors. Approved the final version of the manuscript on behalf of all authors: Thenier-Villa. Statistical analysis: Thenier-Villa. Administrative/technical/material support: Sanromán-Álvarez, Miranda-Lloret. Study supervision: all authors.

\section{Correspondence}

José Luis Thenier-Villa: University Hospital Complex of Vigo, Spain. jose.luis.thenier.villa@sergas.es. 